\title{
Remodelling and Treatment of the Blood-Brain Barrier in Glioma
}

\author{
Yihao Wang (D) \\ Fangcheng Zhang' \\ Nanxiang Xiong ${ }^{2}$ \\ $\mathrm{HaO} \mathrm{Xu}^{3}$ \\ Songshan Chai' \\ Haofei Wang $\mathbb{D}^{\prime}$ \\ Jiajing Wang ${ }^{\prime}$ \\ Hongyang Zhao' \\ Xiaobing Jiang' \\ Peng Fu (D) \\ Wei Xiang'
}

'Department of Neurosurgery, Union Hospital, Tongji Medical College, Huazhong University of Science and Technology, Wuhan, 430022, People's Republic of China; ${ }^{2}$ Department of Neurosurgery, Zhongnan Hospital, Wuhan University, Wuhan, 43007I. People's Republic of China; ${ }^{3}$ Department of Neurosurgery, General Hospital of the Yangtze River Shipping, Wuhan, 430022, People's Republic of China
Correspondence: Peng Fu; Wei Xiang Department of Neurosurgery, Union Hospital, Tongji Medical College,

Huazhong University of Science and Technology, Wuhan, 430022, People's Republic of China

Tel +86 I5902706600; +86 I366729|375

Fax +862785350819

Email pfu@hust.edu.cn;

xiangwei20@hotmail.com

\begin{abstract}
The blood-brain barrier (BBB) is an essential structure of the central nervous system (CNS), and its existence makes the local internal environment of the CNS a relatively independent structure distinct from other internal environments of the human body to ensure normal physiological and high stability of activities of the CNS. Changes in BBB structure and function are fundamental to the pathophysiology of many diseases. The occurrence and development of glioma are often accompanied by a series of changes in the structure and function of the internal environment, the most significant of which is remodelling of the BBB. The remodelling of the BBB usually leads to changes in the permeability of local microvessels, which provide certain favourable conditions for the occurrence and development of glioma. Meanwhile, the newly generated abnormal blood vessels and the remaining intact regions of the $\mathrm{BBB}$ also hinder the effects of drug treatments. Changes in permeability and structural function often lead to the creation of abnormally functioning vascular regions, which pose further treatment challenges. At present, therapeutic methods for glioma have not achieved satisfactory effects in clinical practice, and emerging therapeutic methods have not yet been widely used in clinical practice. In this review, we summarize the knowledge of remodelling of the $\mathrm{BBB}$ in the glioma environment, the type of changes that occur, and current BBB treatment methods and prospects for the treatment of glioma.
\end{abstract}

Keywords: blood-brain barrier, glioma, blood-brain barrier-associated therapy, remodelling, structural and functional changes

\section{Introduction}

In 1885, Paul Ehrlich performed a series of innovative experiments using intravenous injection of aniline dye in experimental animals, which ultimately led to the discovery of the blood-brain barrier (BBB). ${ }^{1}$ For more than 100 years, our understanding of the $\mathrm{BBB}$ has continued to become more profound.

The BBB is composed of the continuous capillary endothelium and tight junctions (TJs) between the cells, the intact basement membrane, the pericytes, the microglia, and the glial membrane surrounded by astrocytes. ${ }^{2}$ The normal BBB structures is shown in Figure 1.

Endothelial cells overlap each other and are tightly connected, effectively preventing macromolecular substances from passing through the junction of endothelial cells. ${ }^{3}$ A continuous basement membrane also surrounds these endothelial cells. On the basement membrane's outer surface are many astrocytes with a perivascular foot (final foot) that surrounds approximately $85 \%$ of the brain capillaries' surface and provides connections to neurons. ${ }^{4}$ The basal lamina, the abluminal surface of the endothelium, consists of cell type IV collagen, fibronectin, 


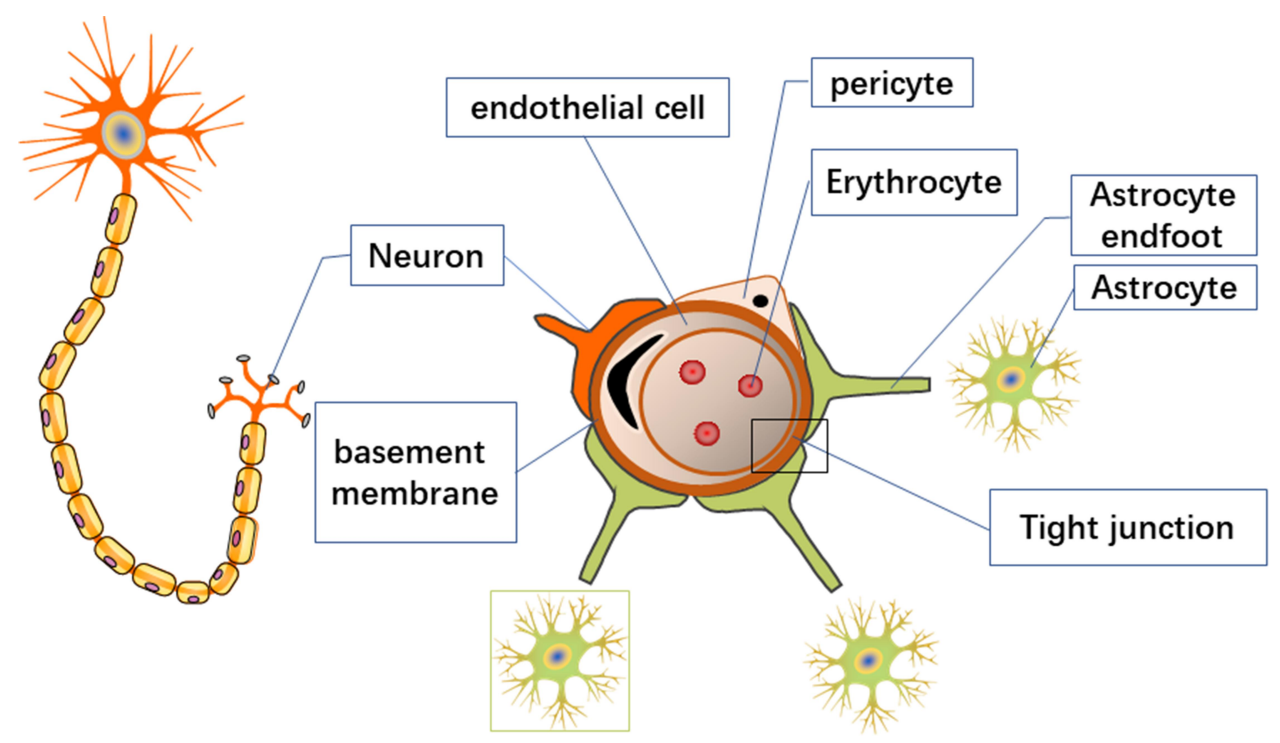

Figure I The normal BBB.

heparin sulphate, and laminin, which form the molecular and charge barrier. $^{5-7}$ Compared to endothelial cells in different tissues, endothelial cells of the BBB are unique because they have continuous TJs, a lack of fenestrations, and a meagre extracellular endocytosis rate, which significantly restricts the movement of molecules through the endothelial cell layer., ${ }^{4,8}$.

TJs are multiprotein junctional complexes whose function is to prevent leakage and seal the paracellular pathway. TJs are multifunctional, ranging from forming selective and tightly regulated barriers to acting as bidirectional signalling hubs to coordinate junction assembly and cell polarization, as well as regulate gene expression and cell proliferation. ${ }^{3}$ The low permeability of endothelial cells means that many specific transporters are needed to supply various compounds to the brain. ${ }^{9}$ TJ-associated signalling mechanisms are now firmly linked to the regulation of cell proliferation, polarization, and differentiation.

Astrocytes play an essential role in maintaining the integrity of endothelial cell barrier functions. Astrocytes form a multilayered membrane structure of brain capillaries, which constitutes a protective barrier for brain tissue. ${ }^{10}$ Pericytes cover the outer surface of endothelial cells, promoting $\mathrm{BBB}$ integrity and inducing barrier properties. ${ }^{11}$

BBB characteristics make it difficult for neurotoxic substances, such as viruses, inflammatory factors, and heavy metals, to enter and accumulate in brain tissue, which is of considerable significance for maintaining the normal physiological function of the CNS.

\section{How Does Glioma Remodel the Blood-Brain Barrier? Structural and Functional Changes}

The effects of glioma cells on the BBB include direct effects on the local $\mathrm{BBB}$ and indirect impacts on the distant $\mathrm{BBB}$ through secretion of various substances. These effects result in local structural and functional changes, which are described below.

\section{Structural Changes Associated with Astrocytes}

As a highly invasive tumour, glioma usually metastasizes along pre-existing brain structures, such as nerve tracts, blood vessels, meninges, and white matter tracks. ${ }^{12-14}$ The changes in blood vessels in glioma include changes in existing blood vessels and the promotion of angiogenesis. Glioma cells attach to blood vessels through bradykinin, produced by vascular endothelial cells, which acts as a chemotactic signalling peptide. ${ }^{15}$ Prior to angiogenesis, glioma tends to encase the outside of the original blood vessels by invasion and parasitism, invading the surrounding space. ${ }^{16,17}$ This invasive behaviour blocks the connection between astrocyte end feet and endothelial cells, which leads to breakdown of the BBB. ${ }^{12}$ Astrocyte end feet and pericytes work together to release large amounts of vasoactive molecules from the junction end to endothelial cells, which regulate vascular tension and help maintain expression of TJ proteins, transporters, and enzymes by releasing soluble factors and maintaining mutual contact. ${ }^{4,18-20}$ However, in response to glioma-induced displacement, these functions are disrupted, while astrocytes alter their phenotype. ${ }^{12}$ At 
the same time, glioma cells can physically move the astrocytic end feet away from the basement membrane of endothelial cells and into the abluminal side of vessels, which also prevents vasoactive substances from reaching endothelial cells. ${ }^{12}$ These changes usually cause degradation of the basement membrane and loss of TJs, leading to serum leakage into the parenchyma. ${ }^{14}$

As the glioma grows, glioma cells migrate along the blood vessels and compress and destabilize the vessels, resulting in vascular degeneration and reduced perfusion. Moreover, these actions can lead to hypoxia and necrosis. Hypoxia and mutations in glioma cells upregulate the secretion of growth factors, such as vascular endothelial growth factor (VEGF), basic fibroblast growth factor (BFGF), interleukin 8 (IL8), and stromal cell-derived factor-1 (SDF1), which generate new blood vessels through angiogenesis. ${ }^{21}$ However, increased expression often leads to abnormal angiogenesis and dysfunctional blood vessels, which creates an adverse microenvironment (reduced oxygen partial pressure and increased interstitial fluid pressure), leading to a malignant phenotype and increased morbidity and mortality. ${ }^{22,23}$ Meanwhile, hypoxia-induced transcription factors recruit activated pericytes to glucose uptake sites from afar. $^{23}$ These structural changes are depicted in Figure 2.

Interestingly, in tumour cells adjacent to blood vessels, intercellular connections and the basal lamina remain. Studies have demonstrated that in the case of U87 cell transplantation, glioma cells can remove the end feet of astrocytes. However, multiple interactions were observed in glioma stem-like cell (GSC) transplantation from
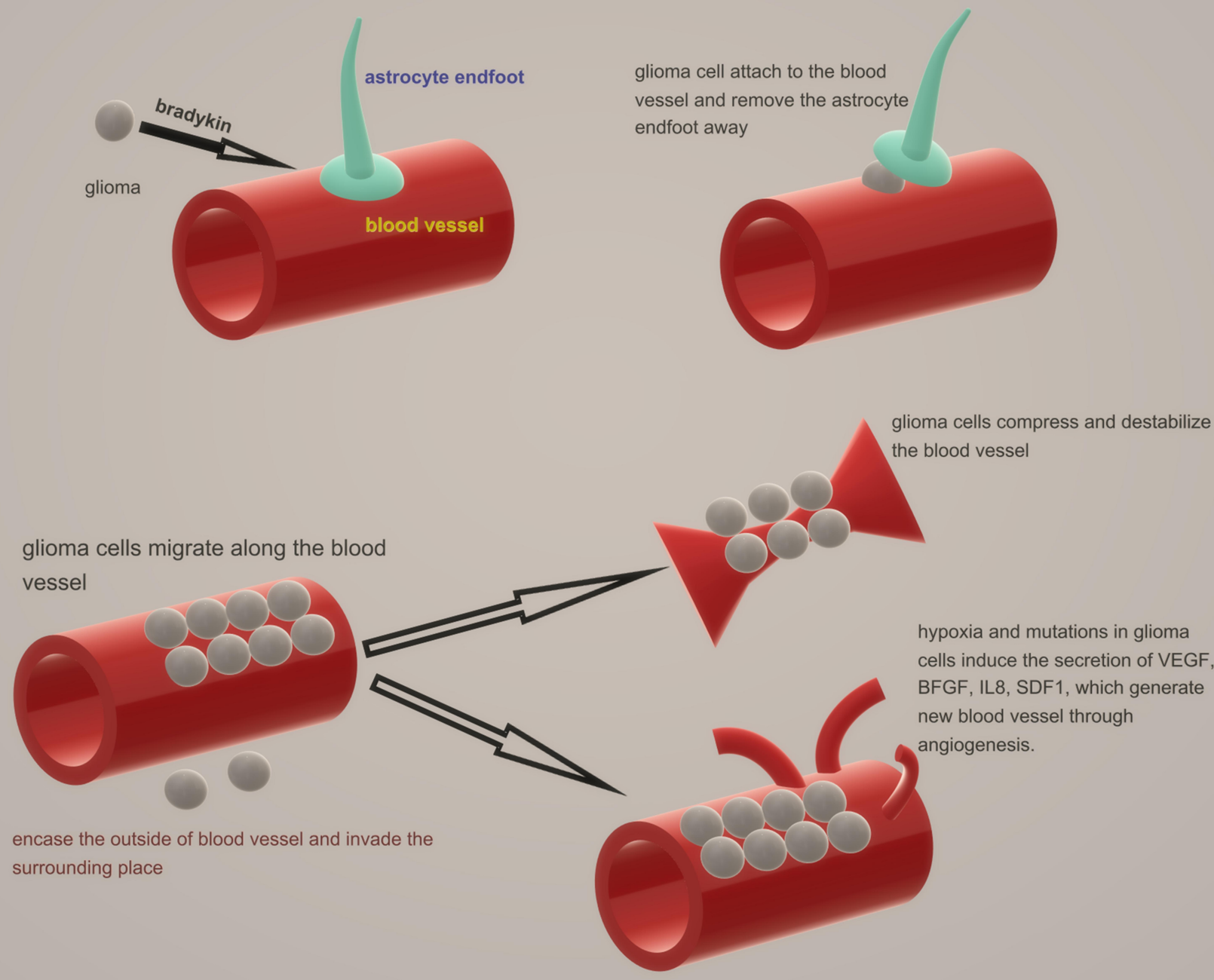

Figure 2 Structural changes in BBB. 
patient samples, and the BBB maintained its integrity. This condition may be related to the molecules carried by serum in different media. ${ }^{24}$

\section{Cytoskeleton and Tight Junctions}

Several studies have demonstrated a close relationship between decreased glial fibrillary acidic protein (GFAP) and s-100 expression in BBB TJs caused by hypoxia/ ischaemia and increased BBB vascular permeability. ${ }^{25}$ GFAP is an acidic protein and the primary cytoskeletal protein of glial cells. S-100 is an acidic calcium-binding protein primarily found in astrocytes in various parts of the central nervous system. It is generally acknowledged that when central nervous system cells are damaged, s-100 protein is extruded into the cerebrospinal fluid from the cell fluid and then enters the blood through the damaged BBB.

TJ structure is primarily composed of transmembrane proteins, such as occludins, claudins, and junctional adhesion molecules (JAMs), as well as peripheral proteins, such as zonula occludens ( $\mathrm{ZO}$ ). On the one hand, the structure of this functional barrier mediated formed by complex compounds restricts the diffusion and transport of ions, water, and other solutes in the paracellular space. On the other hand, it limits the random diffusion of membrane proteins and lipids on the top and basal sides of the plasma membrane, maintains cell polarity, and acts as a fence. ${ }^{26,27}$

In the extracellular loop (EL), occludins form dimers with adjacent cells and build TJs in the intercellular space. Occludins participate in endothelial cell differentiation and signal transduction. Upregulation of VEGF expression due to hypoxia and necrosis can activate the protein kinase $\mathrm{C}$ (PKC) signalling pathway, leading to phosphorylation of occludins and affecting endothelial cell permeability, ${ }^{28}$ which destroys the local structure of the BBB.

Claudins play an important role in maintaining BBB structure and functional stability. Meanwhile, claudins are considered the main $\mathrm{TJ}$ transmembrane proteins in the $\mathrm{BBB}$ and are an essential component in the formation of ultrastructures that determine the characteristics of TJs. Abnormal expression of claudins is associated with various CNS diseases, such as brain tumours and Alzheimer's disease. Claudin-5 is a cytoskeletal protein that makes up the TJs between endothelial cells. Pathologically, claudin5 can be degraded by matrix metalloproteinase-9 (MMP9), leading to increased BBB permeability, mainly to small molecular substances. ${ }^{29,30}$ Guo et al found that IncRNA
NEAT1 is associated with miR-181d-5p/SOX5 pathway regulation, leading to increased permeability of the BBB by reducing $\mathrm{TJ}$ protein (ZO-1, occludin, claudin-5) expression. ${ }^{31}$ Similarly, miR-34c and miR-18a were reported to have the same functions. ${ }^{32,33}$

The relationship between junctional adhesion molecules and BBB remodelling in glioma conditions is not clearly understood.

ZO includes three subtypes: ZO-1, ZO-2, and ZO-3. The ZO-1 protein contains multiple functional domains and performs its corresponding physiological functions by combining with different proteins. ${ }^{34}$ For example, overexpression of endophilin-1 inhibits ZO-1 and enhances cell bypass permeability through the epidermal growth factor receptor (EGFR)-ERK1/2 and EGFR-JNK pathways. $^{35,36}$

The direct effect of glioma cells on the BBB can significantly reduce expression levels of ZO-1 in ECV304 cells. In contrast, the indirect impact had no significant effect on expression levels of ZO-1 in ECV304 cells. This suggests that expression levels of ZO-1 are a crucial molecular mediator for the impact of glioma cells on TJs of the BBB. ${ }^{12,37-39}$ Decreased or absent expression of ZO-1 leads to loss and even decomposition of the TJs that ultimately affects the functional integrity of the BBB, which is the cause of peritumour oedema. Lin et al found that miR-424-5p may affect $\mathrm{TJ}$ proteins (ZO-1 and occludin) through endorphin-1, regulating BBB permeability in a BBB model of endothelial cells incubated with Abeta in vitro. ${ }^{40}$ The increased microvascular permeability in human glioma results in severe clinical cerebral oedema due to the imbalance in junctional proteins. $^{41}$

\section{Extracellular Matrix}

The extracellular matrix in the tumour microenvironment, including collagen IV, fibronectin, heparin sulphate, and laminin, plays a vital role in glioma invasion and progression. Gliomas often exhibit necrotic areas. As a result, hyaluronic acid in the extracellular matrix can activate the white blood cell toll-like receptors (TLR) pathway, secrete tumor necrosis factor (TNF) and transforming growth factor- $\beta$ (TGF- $\beta$ ) and regulate matrix metalloproteinase (MMP) expression and chemokines, thereby activating the immune system and remodelling the extracellular matrix. ${ }^{42}$ Laminin is a noncollagen sugar that stabilizes the vasculature and keeps $\mathrm{T}$ lymphocytes out of the brain through integrin $\alpha 6 \beta 1$ to maintain CNS 
stability and contribute to endothelial junctional tightness. ${ }^{43,44}$ Savettieri et al reported that collagen IV can increase endothelial cell TJ claudins mRNA expression. ${ }^{45}$ In laminin gene knockout mice, plateletderived growth factor receptor- $\beta$ (PDGFR- $\beta$ ) and contractile protein expression in pericytes were upregulated, the basal membrane of pericytes was fragmented and dispersed, the fluorescence intensity of Evans blue staining in the brain parenchyma was higher than in the control group, and expression of transporters AQP4, ligandin, occludin, and claudin-5 was downregulated. ${ }^{46}$ Meanwhile, glutamate concentration was increased in the extracellular fluid of the BBB basal surface, and excessive glutamate release enhances vascular permeability. ${ }^{47,48}$

\section{Transendothelial Electrical Resistance and the Endothelial Barrier}

Previous studies have shown that the permeability of endothelial cells is related to their transmembrane resistance. Astrocytes induce endothelial cells to form TJs and produce higher transendothelial electrical resistance (TEER). ${ }^{28}$ These TEER values can be increased by the extent of the parietal endothelial disruption. Meanwhile, changes in TJ proteins can also alter TEER values.

However, in response to the formation and action of glioma, TEER can be significantly reduced because TJs between endothelial cells become compromised, and residual TJs can occasionally be seen in some areas. ${ }^{49}$

Mendes et al found that during the coculture of glioma cell lines and endothelial cells, decreased expression of claudin-5, occludin, and vascular endothelial cadherin (VE-cadherin, CD144) markers was observed by immunofluorescence, leading to the destruction of tight connections. Meanwhile, a decrease in the paracellular values of TEER was also observed. ${ }^{49}$

\section{Cellular and Molecular Changes Pericytes}

Pericytes express a variety of $\mathrm{TJ}$ proteins and transporters. ${ }^{50}$ Pericytes and endothelial cells secrete TGF- $\beta 1$ through the PDGF/PDGF- $\beta$ pathway to inhibit vascular cell migration, maintaining $\mathrm{BBB}$ integrity. Pericytes maintain vascular integrity by negatively regulating fibrinolysis of endothelial cells, regulating BBBspecific gene expression pattern in endothelial cells, and inducing polarization of astrocyte end-feet surrounding CNS blood vessels. ${ }^{51-53}$ In PDGF knockout mice with pericyte dysfunction, the permeability of the BBB to water and small molecular weight tracers was significantly increased. ${ }^{53,54}$ Furthermore, expression of the TJ proteins ZO-1 and occludin in PDGF- $\beta$ transgenic mice was downregulated. ${ }^{55}$ The proliferation of pericytes and the distribution of tumour vessels are associated with Notch signalling. The absence or mutation of Notch3 inhibits the expansion of pericytes, leading to decreased BBB integrity. ${ }^{56}$

Tissue damage and the multimodal function of pericytes may be related to glioma proliferation, resulting in activation or damage. When gliomas proliferate and invade, the vascular supply and the expression of growth factors required by cells are increased. Therefore, pericytes are highly expressed in proliferating tissues with oedema and inflammation, which characterizes the glioma microenvironment. ${ }^{57}$ Under hypoxic conditions, pericytes maintain mitochondrial activity, thus maintaining the integrity of TJs. ${ }^{58}$ However, in the case of chronic disease, the damage or loss of pericytes caused by hypoxia and hypoperfusion leads to a reduction in BBB integrity and vascular stability. ${ }^{59}$ Amyloid beta (A $\beta$ ) deposition can lead to the loss of pericytes under the same conditions. Interestingly, it was reported that pericyte-associated neurotrophic factors can increase the expression of claudin-5 in cerebral microvascular endothelial cells and TEER level of cerebral microvascular endothelial cells, enhancing the barrier function of the BBB. ${ }^{60}$

Glioma-associated mesenchymal stem cells (gaMSCs) have been proven to transform into pericytes under certain conditions. ${ }^{61,62}$ These findings may present new opportunities for treatment strategies. However, many functions of gaMSCs remain to be understood.

\section{Water Channels and Aquaporins}

Aquaporins (AQPs) belong to the water channel family, some of which are also involved in transporting other small molecules, such as glycerol and urea. At the same time, their expression and localization often affect glioma metastasis and angiogenic oedema. Studies have shown that aquaporins are involved in many aspects of glioma pathogenesis, such as promoting the movement and invasion of glioma cells under hypoxia, promoting the formation of oedema, and improving the metabolism of glioma cells during glycolysis. ${ }^{63}$

Aquaporin 4 (AQP4) is the main water channel in the brain and is expressed in various CNS structures. ${ }^{64}$ It is primarily an astrocyte membrane protein that is localized at the end of astrocytes and points to microvessels. At the 
same time, the AQP4 molecule helps to form orthogonal arrays of particles (OAPs), which serve as a critical functional component of the BBB.

Expression of AQP4 in glioma is impaired, and its arrangement is disordered, leading to obstruction of the transmembrane transport function of water molecules. ${ }^{64}$ Astrocyte podocytes induce the formation of TJs of the BBB and highly express AQP4. ${ }^{65}$ The disturbed MMP3/ TIMP-1 balance in glioblastoma may be one reason for the increased agrin degradation. The reduction in agrin was accompanied by the redistribution of AQP4 across the surface of glioma cells. ${ }^{9}$ The loss of agrin and AQP4 leads to loss of cellular polarity and consecutive oedema formation. ${ }^{66,67}$ Zhou et al found that brain microvessel ultrastructure in mice without AQP4 is often altered, including open TJs and astrocyte swelling around blood vessels, hyperpermeability of the BBB, and glial fibrillary acidic protein (GFAP) immunoreactivity. ${ }^{68}$

Interestingly, Saadoun et al found that the absence of AQP4 in mice did not alter the integrity of the BBB or brain morphology, ${ }^{69}$ while Zhang et al observed increased capillary density and decreased water exchange in the $\mathrm{BBB}$ in AQP4-KO mice. ${ }^{70}$ Further research is needed to confirm these findings.

\section{Caveolin}

Caveolin-1 is a significant membrane intrinsic protein in caveolae on the cell surface that plays a specific role in maintaining caveolae integrity, cell transport, and signal transmission. Caveolin-1 contributes to tumour angiogenesis, growth, and metastasis. In glioma, caveolin-1 overexpression induces reduced proliferation, clonogenicity, and migration. ${ }^{71}$ Caveolin-1 can also induce increased BBB permeability. Furthermore, there appears to be heterogeneity in caveolin-1 expression among glioma cells, suggesting that caveolin-1-positive and caveolin-1-negative cells coexist in glioma. ${ }^{72}$ In astrocytes, caveolin-1 binds to multidrug resistance protein-1 (MDR1), also called p-glycoprotein 1 (P-GP), which is a transporter involved in the function of the $\mathrm{BBB}{ }^{73}$

\section{Vascular Endothelial Growth Factor (VEGF)}

Progressive growth of tumour tissue is accompanied by increased VEGF expression, indicating the formation of new blood vessels. Changes in VEGF expression associated with the permeability of the BBB may cause stellate cells to produce a large number of factors that are harmful to the $\mathrm{BBB}$, leading to increased permeability of the BBB and induction of brain oedema in the process of microvascular leakage. Many bioactive cytokines play a role in this process, and increased expression of VEGF is one of the primary factors. ${ }^{74}$ Due to a lack of normal anatomy of the new vascular tissue, BBB permeability increases. Other changes related to VEGF are described above. These changes are illustrated in Figures 2 and 3.

\section{Radiological Changes}

In areas where the BBB has been physically destroyed, hydrophilic contrast agent molecules disperse out of the vascular cavity and gather in the extracellular space outside the blood vessels. Almost all T1-weighted (T1W) sequences of glioblastoma (GBM) show a high-intensity region of enhanced contrast. ${ }^{75}$ However, MR and PET imaging demonstrate that most GBMs have an intact BBB that extends beyond the contrast-enhancing tumour volume. ${ }^{76}$ This result indicates that the BBB in GBM is not entirely destroyed, and all GBMs have some areas of intact $\mathrm{BBB}^{76}$

\section{The Effect of Remodelled BBB on Glioma}

Destruction of the BBB leads to molecular extravasation of the serum, which results in apparent cerebral oedema, nodular tumour growth, satellite nodules, and tumour invasion.

Changes in the BBB are independent of the size, type, and anatomical location of the glioma. ${ }^{11}$ Compared to lowgrade glioma, high-grade glioma was significantly different, with low-grade glioma exhibiting no distinct changes. ${ }^{77}$ Meanwhile, a single invading tumour cell that removes the astrocyte tip from the endothelial cell is enough to cause a local rupture of the BBB. ${ }^{12}$

Initially, when the $\mathrm{BBB}$ function is damaged, the most common result is vasogenic brain oedema, which is caused by many large molecular weight substances in the plasma entering the extracellular fluid of brain tissue so that the water passively diffuses into the brain tissue gap through the BBB to cause brain oedema.

Furthermore, when glioma cells act on the BBB, vascular endothelial cells proliferate abnormally, promoting tumour neovascularization, which may further accelerate the occurrence and development of glioma.

Finally, changes in the BBB may prevent effective treatment by glioma drugs. Due to the varying degree of damage to the BBB in different regions, factors such as the 


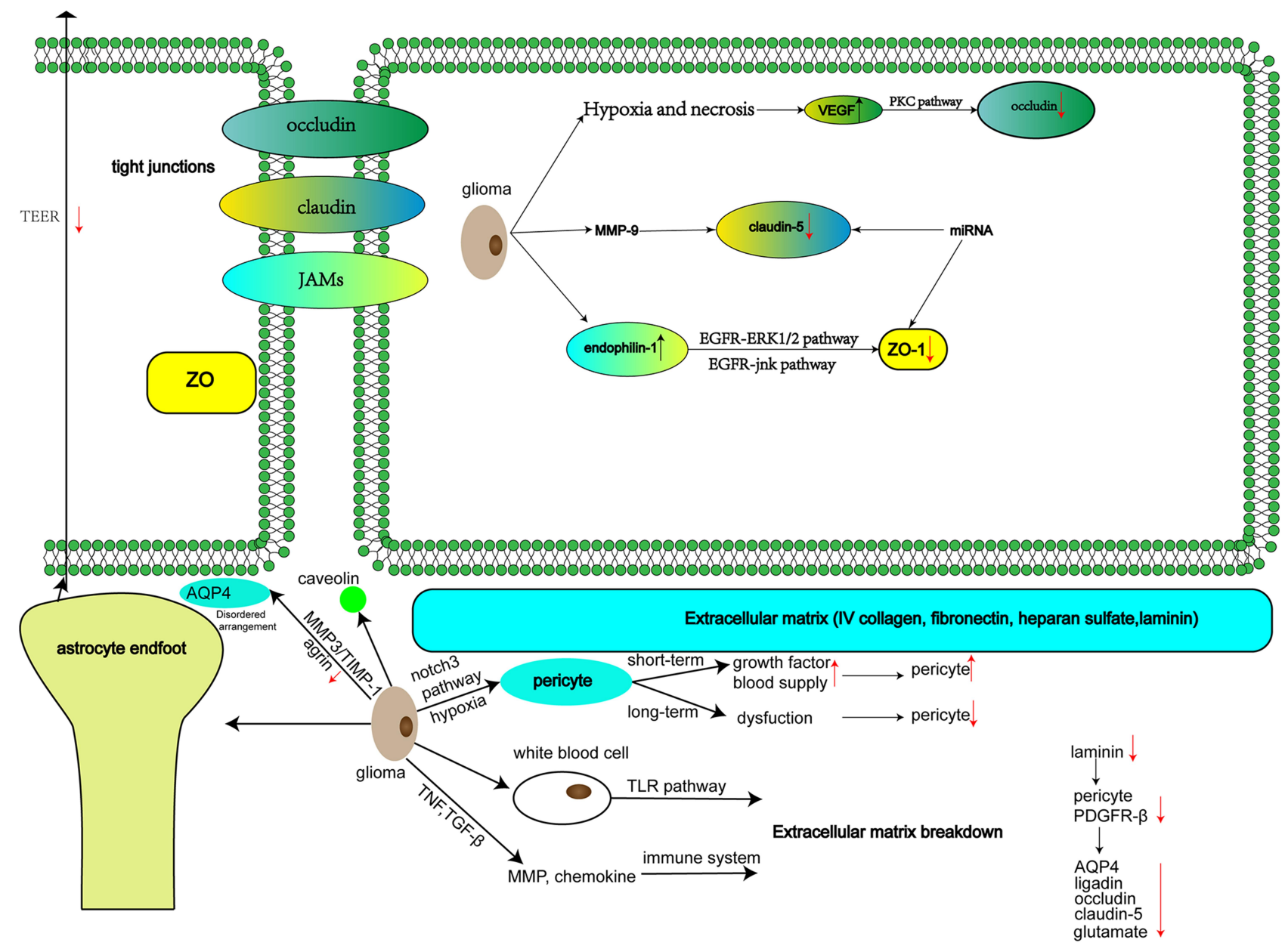

Figure 3 Cellular and molecular changes.

dosage, administration method, and specificity of drugs should be carefully considered. Therefore, these changes convey some difficulties in postoperative clinical treatment.

\section{Treatments with Respect to the Blood-Brain Barrier}

The invasive and aggressive nature of glioma makes complete surgical removal impossible, while the BBB prevents most treatments from reaching the remaining tumour cells. Therefore, metastatic and residual tumour cells need to be treated with therapies that penetrate the BBB. The physiological protection provided by the BBB makes the delivery of drugs to the brain challenging, which affects the central nervous system. The most common way to ensure that drugs pass the $\mathrm{BBB}$ is to use existing channels or to create a new pathway.

See Figure 4 for the schematic diagram of the overall treatment, and figure legend for details.

\section{Associations of Tight Junctions and Therapeutic Methods Hypertonic Solutions and Bradykinin}

Hypertonic solutions can destroy the BBB instantaneously, leading to contraction of endothelial cells, widening the gap between TJ proteins, and promoting diffusion of drug molecules into the brain. ${ }^{78}$ Ma et al found that Krüppellike factor 4 (KLF4) regulates the permeability of the blood-brain tumour barrier (BTB) by regulating the expression of the tightly bound and related proteins ZO1 , occludin, and claudin-5 and may be a key transcriptional regulator of BTB function. ${ }^{79}$ The BTB is similar to the BBB and is located between glioma cells and microvessels formed by highly specialized endothelial cells. In vivo, animal studies have shown that vasoactive substances, such as bradykinin, histamine, bradykinin analogues, and RMP-7, stimulate B2 receptors on endothelial cells, instantly increasing the intracellular $\mathrm{Ca}^{2+}$ concentration, activating nitric oxide synthase, and increasing nitric 

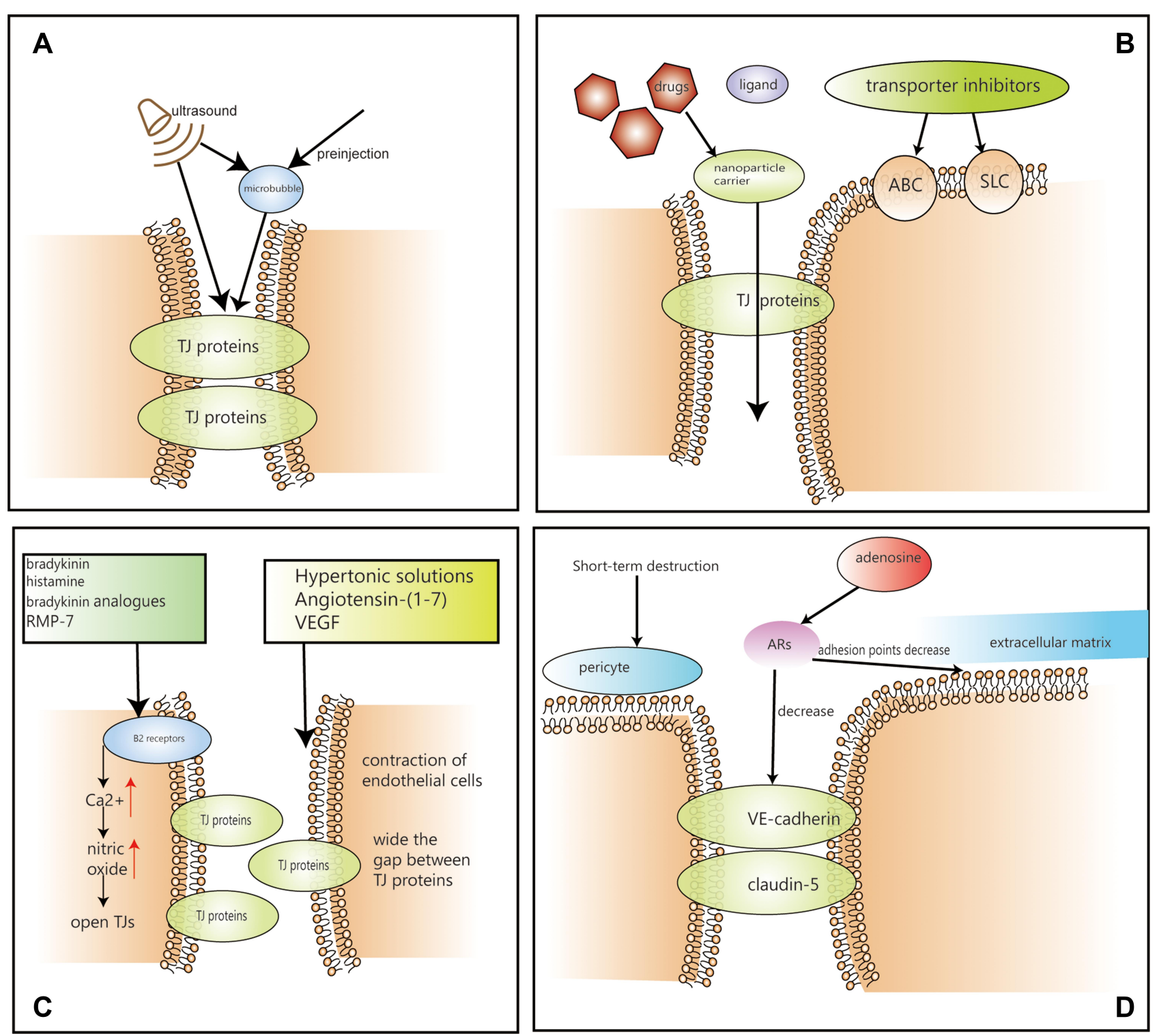

Figure 4 Treatments based on BBB (A) The process of ultrasonic treatment. (B) The process of nanoparticle carrier delivery system and transporter system. (C) The process of bradykinin, hypertonic solution, VEGF and angiotensin-(I-7). (D) The process of short-term destruction and adenosine.

oxide concentration, thereby dilating blood vessels and opening TJs. ${ }^{80,81}$ However, the infusion of hypertonic solutions, such as mannitol, is not widely used in clinical practice as it constitutes an invasive treatment that requires close coordination. Other methods of bradykinin modifying TJs have not been clinically proven to be effective.

\section{Adenosine Receptors}

Adenosine receptors (ARs) have four different subtypes, $\mathrm{A} 1, \mathrm{~A} 2 \mathrm{~A}, \mathrm{~A} 2 \mathrm{~B}$, and A3. ${ }^{82}$ Human endothelial cells are capable of producing and responding to adenosine. Moreover, activated A2A AR effectively increases the permeability of the $\mathrm{BBB}$ in vitro in a reversible manner, which is essential for patient safety. ${ }^{83}$ Thus, adenosine can be used to improve BBB permeability by activating A2A AR to decrease the expression of VE-cadherin and claudin-5. Additionally, adhesion points between endothelial cells and their extracellular matrix were decreased, RhoA activity was increased, and actin stress fibres formed. ${ }^{84}$ These studies suggest that regulation of the BBB by AR signalling may be a feasible strategy for the delivery of therapeutic drugs to glioma, with fewer side effects. However, this method has not entered the clinic. Nevertheless, clinical evaluation is expected.

\section{Angiotensin-(1-7)}

Angiotensin-(1-7) is a bioactive fragment of the reninangiotensin system (RAS). Studies have shown that Ang- 
(1-7) might reduce the destruction of the BBB in a glioma model in rats by activating expression of $\mathrm{TJ}$ proteins in endothelial cells through downregulating MMP-9. ${ }^{85}$ Meanwhile, the c-Jun N-terminal kinase (JNK) pathway is involved in the mediating effect of Ang-(1-7). SP600125 is an inhibitor of the JNK pathway that can significantly enhance the expression of claudin-5 and ZO-1 and reduce JNK pathway interference with claudin5 and ZO-1. Therefore, Ang-(1-7) might be a therapeutic target due to its ability to affect BBB permeability, tumour growth, and oedema formation. ${ }^{86}$

\section{Short-Term Destruction Method}

Using drugs to destroy glioma stem cell-derived pericytes in a short time, the reduction of $\mathrm{TJ}$ proteins and the destruction of TJ structures on glioma vessels can increase vascular permeability without affecting tumour vascular density, promoting the delivery of chemotherapy drugs. ${ }^{87}$ Alvarez et al applied the Smoothened inhibitor cyclopamine to disrupt TJs in the brains of normal rats, demonstrating that the in-depth study and utilization of signalling pathways that maintain the stability and integrity of the BBB may represent several methods and pathways that can be exploited for drug delivery into the brain. ${ }^{88}$

\section{VEGF}

VEGF and smoothed signalling pathways play an important role in maintaining the stability of $\mathrm{TJ}$ proteins. ${ }^{88}$ It was found that interfering with these signalling pathways can cause TJ proteins to lose stability, subsequently improving BBB permeability. ${ }^{89}$ VEGF downregulates or retargets the $\mathrm{TJ}$ protein claudin-5, leading to instability in TJs and increasing BBB permeability. Wen et al found that interfering with the VEGF/VEGFR pathway through use of the inhibitor axitinib reduces $\mathrm{BBB}$ permeability and enhances integrity of the barrier. Simultaneously, lowdensity lipoprotein receptor-related protein 1 (LRP1) is highly expressed in the BBB under pathological conditions. Therefore, doxorubicin (DOX)-loaded glycolipidlike nanoparticles (AP-CSSA/DOX) loaded into the BBB and low-density lipoprotein receptor expressed in GBMassociated protein 1 (LRP1) have a high affinity for the tumour and achieve good antitumour effects. ${ }^{90}$ Bevacizumab specifically binds to VEGF (primarily VFGF-A), attenuating or blocking VEGF binding to VEGFR-1 and VEGFR-2 on the vascular endothelial cell surface and blocking VEGFR-mediated downstream signalling pathways. ${ }^{91}$ However, current clinical trials show that bevacizumab has not achieved good, predetermined efficacy in improving long-term outcomes in patients with GBM. This phenomenon may be related to the incomplete understanding of the upstream and downstream mechanisms of VEGF.

\section{Ultrasonic Treatment-Associated Effects}

Specifically, microbubbles 2-6 microns in diameter formed by preinjection can be induced by ultrasound, causing the $\mathrm{BBB}$ to open instantaneously, which can be monitored by MRI or ultrasound. ${ }^{92}$ Zhang et al found that ultrasound-bound microbubbles could open the BBB through paracellular and transcellular pathways. They primarily cause changes in the expression of BTB endothelial cell membrane TJ proteins, such as claudin-5, ZO-1, and occludin, reduce the expression of JAM-A and upregulate $\mathrm{KCa}$ channel expression in glioma. ${ }^{93}$

Transient disruption of the BBB by focused ultrasound (FUS) facilitates noninvasive drug delivery with precise spatial and temporal specificity. ${ }^{94}$ Chen et al found that focused ultrasound improves the antitumour immune response of interleukin-12 (Il-12) in c-6 glioma rats, safely and reversibly disrupting the $\mathrm{BBB} .^{95}$ Ting et al used focused ultrasound combined with microbubble technology to improve carmustine penetration in the rat brain. ${ }^{96}$ This method increases local drug concentrations in glioma to promote treatment more effectively.

Zhang et al found that low-frequency ultrasound (LFU) and bradykinin (BK) caused increased BTB permeability by regulating the expression of the TJ-related proteins $\mathrm{ZO}-$ 1, Occludin, and Claudin-5. ${ }^{97}$ Wang et al found that lowfrequency ultrasound irradiation combined with the use of papaverine causes a similar effect. ${ }^{98}$

Ultrasound-related therapy has only been used in preclinical studies, and its ability to open the tumour BBB is still being explored. However, ultrasound-related treatment is considered a promising method that facilitates transient, localized, and noninvasive changes in the brain for drug administration. It has broad applications in the future for use in combination with newly developed drugs.

\section{Transportation-Associated Effects}

BBB endothelial cells contain a variety of transporters through which drugs travel from the brain to the blood. ${ }^{99}$ Two major protein families drive carrier-mediated transport, the solute carrier (SLC) superfamily and ATP binding cassette $(\mathrm{ABC})$ transporters, which prevent the influx of lipophilic molecules, xenobiotics, toxic metabolites, and 
drugs. ${ }^{100}$ It has been reported that there are also extraneous transporters on GBM cells, forming the brain-tumour cell barrier, while the BBB and brain-tumour cell barrier make it more difficult to transport drugs to glioma tissues. ${ }^{96}$ Chen et al discovered a peptide from phages that can be used as a generic carrier to cross the BBB. ${ }^{101}$ Agarwal et al found that the combined use of elacridar increased the concentration of erlotinib in the brain of a xenograft model of glioma in U87 rats. ${ }^{102}$ Therefore, efflux transporter inhibitors are an important method for promoting drug transport into the brain.

Recent studies have shown that phosphatidylinositol-3 kinase inhibitors, which mediate cell proliferation, apoptosis, metabolism, angiogenesis, and other biological processes, promote GBM cell death and have good prospects for the treatment of GBM. ${ }^{103}$ Currently, many drugs that inhibit efflux transporters or utilize existing transporters are undergoing clinical trials with promising results.

$A$ variety of $\mathrm{ABC}$ transporters, such as P-glycoprotein (P-GP), multidrug resistance associated protein (MRP), and breast cancer resistance protein (BCRP), are efflux transporters that are highly expressed at the BBB, which are also the primary factors inhibiting introduction of drugs into the brain, which is the primary reason for the poor efficacy of commonly used antitumour drugs against glioma $.99,104$ Meanwhile, studies have found that the BBB of glioma patients is destroyed due to the influence of pathological factors. However, there is still an intact $\mathrm{BBB}$ in the glioma tumour area, preventing active drug ingredient distribution to tumour cells. ${ }^{24,105}$ Interestingly, the more significant increase in permeability compared to the sum of the two transporters was due to functional compensation on the BBB between P-GP and BCRP rather than simply upregulation of transporters. ${ }^{102}$

Convection-enhanced delivery (CED) is the continuous delivery of a drug by an implanted infusion catheter during surgery that relies on a hydrostatic pressure gradient to enable the drug to reach the tumour cell through substantial convection. The drug will be widely distributed in the brain by dispersing large quantities. However, this method's challenge is that medicines injected into the brain cannot easily spread or transport into the blood, making the drug concentration at the tumour site too low to achieve the desired effect. ${ }^{106}$

Liposomes are vesicles formed by the self-assembly of amphiphilic phospholipids. The structure of liposomes is similar to the lipid bilayer of the cell membrane. Liposomes can embed water-soluble drug molecules and lipophilic drug molecules to facilitate transport to the corresponding target sites. ${ }^{107}$ The biocompatibility and targeting characteristics of liposomes can effectively promote drug transport across the BBB, improving the therapeutic index of chemotherapeutic drugs and reducing adverse reactions. Tumours targeting polymer checkpoint inhibitors can activate systemic or local tumour immune responses through the $\mathrm{BBB}$, playing a therapeutic role. ${ }^{108}$ Based on peptide research, it has been shown that cell penetrating peptides (P1NS) and an anti-GBM antibody (TN-C) can bind to the surface of liposomes for targeted delivery. Superparamagnetic iron oxide nanoparticles (SPIONs) and doxorubicin (DOX) are coloaded in liposomes to achieve thermally triggered drug release when an alternating magnetic field (AMF) is applied. These results show that the structure can be easily transported through the in vitro BBB model. ${ }^{109}$

\section{Nanoparticle Carrier Delivery System-Associated Effects}

Polymer nanoparticles have many of the same advantages as drug carriers, such as many functional groups on the surface of the polymer, which can bind a variety of biological molecules. Commonly used polymer nanoparticle carrier materials include chitosan, dextran, polyethylene glycol, poly(lactic acid)-hydroxyacetic acid copolymer, and dendrimer, which can deliver antitumour drugs to glioma cells through polymer nanoparticles. ${ }^{110}$ Although nanoparticles remain in tumour cells for a long time and are easily cleared by the reticuloendothelial system, they have an uncertain plasma circulation time, but ligand modification of nanoparticles can reduce toxicity and promote drug delivery to the brain. ${ }^{107}$

The most classic example is the use of doxorubicin in the treatment of breast cancer, which has been studied for intracerebral administration due to its high rate of brain metastases. Nanomodified doxorubicin exhibited better results than the original doxorubicin ${ }^{111}$ because lipid molecules are free to spread through the BBB. Research has indicated that thermoresponsive lipid nanoparticles (LNPs) might be an effective treatment because of their natural tendency to cross the BBB. LNPs undergo a solid-liquid phase transition at $39^{\circ} \mathrm{C}$, and as the drug rapidly diffused from the liquid nanoparticles, it provided up to 20 times the drug release rate without any damage to the BBB. ${ }^{112}$ However, the relatively harsh reaction conditions may limit the use of this method.

Mesoporous silica nanoparticles (MSNs) were customized and coupled with the cRGD peptide to be loaded 
with the antitumour drug doxorubicin, which showed a strong ability to penetrate the blood-brain barrier and destroyed the vasculogenic mimicry ability of glioma cells to achieve an excellent antitumour effect, avoiding adverse toxicity and side effects on normal brain tissue. ${ }^{113}$ Albumin nanoparticles, the cell-penetrating peptide LMWP, can achieve glioma cell apoptosis and antiangiogenesis through SPARC- and gp60-mediated biomimetic transport mechanisms and adjust the tumour immune microenvironment to achieve a therapeutic effect with fewer adverse reactions. ${ }^{114}$ Fan et al found that human h-ferritin (HFn) nanocarriers can successfully kill glioma cells by crossing the BBB through the overexpressed HFn receptor (transferrin receptor 1) in endothelial cells and glioma cells. ${ }^{115}$ Meanwhile, there was no HFn buildup in the surrounding healthy brain tissue. Therefore, HFn is an ideal nanocarrier for the treatment of glioma with excellent potential. Similarly, nanoparticles made of lactoferrin (Lf) protein have been shown to enhance the pharmacological properties of drugs. ${ }^{116}$ Large-scale clinical trials of nanoparticles could facilitate the future clinical use of these methods. The specific functions of other extracellular vesicles (EVs), such as exosomes, have been described in detail in a review by Rufino-Ramos et al. ${ }^{117}$

Kolter et al prepared ester-loaded nanoparticles modified with Tween-80 surfactant and treated endothelial cells with reduced TEER. ${ }^{118}$ However, nanocrystalline silver can damage TJs of endothelial cells, causing cell gap widening, cell cavitation, organelle necrosis, coarse endoplasmic reticulum degranulation, depolymerization, and mitochondrial swelling. ${ }^{119}$ Therefore, the potential toxicity of nanomaterials should be closely monitored when they are used.

Nanomaterials show promise, and several experiments have demonstrated different methods for crossing the BBB and delivering therapeutic drugs to tumour cells with fewer toxic side effects. However, there is no large-scale clinical use of nanomaterials at present, and their surface properties, size, and targeting properties should be carefully considered before entering clinical use. The high cost of nanomaterials is also an issue that needs to be addressed.

\section{Current Status and Deficiencies in Treatment of the BBB}

The physical and biochemical barriers of the BBB limit the transport of many drugs into the brain, representing one of the bottlenecks of glioma treatment. Glioma recurrence often occurs in areas far from the edge of the surgical resection, indicating that the drug concentration of glioma cells in these areas was low and thus not adequately treated. It also indicates that the BBB near the growth edge of the glioma was intact. However, because of the high invasiveness of glioma, recurrence easily occurs in the residual area. Currently, the drugs in use, whether chemotherapy drugs, immunotherapy or monoclonal antibodies, are all subject to the presence of residual $\mathrm{BBB}$ and perfusion differences in tumour vessels, resulting in different drug concentrations in various sites, which affects their clinical efficacy. Currently used drugs, such as bevacizumab and cilengitide, are still in clinical trials. Although bevacizumab has been shown to extend progression-free survival as an anti-angiogenesis target drug in previous trials, it does not extend overall survival and has a tendency to promote tumour cell invasion and migration. Based on the above issues, current treatment should focus on the following points. First, many approaches to improving glioma drug delivery are still in the experimental and preclinical stages, with only a few entering clinical trials. For those methods that have already passed preclinical trials, the pace of clinical trials should be accelerated under proper supervision. Furthermore, this area's main challenge is the limited concentration of drugs in glioma since transversely passing drugs through the BBB represents a significant barrier. Therefore, the current focus remains improving the pass rate through various methods to achieve higher effective drug concentrations. Treatment of the residual intact blood-brain barrier and more comprehensive inhibition of angiogenesis should also be considered. Bevacizumab is a VEGF-targeted drug, so the upstream and downstream molecules that simultaneously control VEGF expression and regulation can be considered potential therapeutic targets. Finally, safety remains a significant concern regarding these treatments. ${ }^{120}$

\section{Summary and Future Directions}

Brain tumours, such as glioma, offer significant challenges to neurosurgery and related disciplines. Generally, the treatment methods for glioma primarily include surgical therapy, radiotherapy, chemotherapy, immunotherapy, gene therapy and molecular targeted therapy. After partial treatment, tumour volume is reduced but not completely controlled, and local adjuvant gamma knife therapy may be considered. However, the survival time of glioma patients, especially glioblastoma patients, is still relatively short. Under glioma conditions, remodelling of the BBB 
leads to abnormal neogenesis of blood vessels and a residual intact blood-brain barrier, which brings new challenges to treatment. With in-depth research on the biological characteristics of glioma, many molecularly targeted drugs have been developed, which are more specific than traditional chemoradiotherapy and have a significant inhibitory effect on the invasiveness of glioma. However, some medications require additional support to work at the appropriate sites because of their properties. Current research in this direction is investigating glioma treatment, which is the main direction of clinical research. At present, emerging technologies, such as nanotransport particles, transporters, ultrasound, and other methods have achieved some results in studies on the passage of chemotherapy drugs and targeted drugs through the BBB. However, most of these methods are still in the preclinical research state and have not yet entered clinical use. Therefore, the future research direction should be a combination of new technologies and traditional methods to improve the permeability of the $\mathrm{BBB}$, promote the entry of drugs into the brain as much as possible, and reduce the toxic effect on normal cells, which is the biggest challenge of future research endeavours.

\section{Abbreviations}

BBB, blood-brain barrier; TJs, tight junctions; GBM, glioblastoma; CNS, central nervous system; VEGF, vascular endothelial growth factor; GSC, glioma stem-like cells; GFAP, glial fibrillary acidic protein; JAMs, junctional adhesion molecules; ZO, zonula occludens; TEER, transendothelial electrical resistance; AQP4, aquaporin 4; ARs, adenosine receptors; CED, convection-enhanced delivery; gaMSCs, glioma-associated mesenchymal stem cell.

\section{Disclosure}

The authors report no conflicts of interest in this work.

\section{References}

1. Hawkins BT, Davis TP. The blood-brain barrier/neurovascular unit in health and disease. Pharmacol Rev. 2005;57(2):173-185. doi:10.1124/ pr.57.2.4

2. Sweeney MD, Zhao Z, Montagne A, Nelson AR, Zlokovic BV. Bloodbrain barrier: from physiology to disease and back. Physiol Rev. 2019;99(1):21-78. doi:10.1152/physrev.00050.2017

3. Zihni C, Mills C, Matter K, Balda MS. Tight junctions: from simple barriers to multifunctional molecular gates. Nat Rev Mol Cell Biol. 2016;17(9):564-580. doi:10.1038/nrm.2016.80
4. Abbott NJ, Ronnback L, Hansson E. Astrocyte-endothelial interactions at the blood-brain barrier. Nat Rev Neurosci. 2006;7 (1):41-53. doi: $10.1038 / \mathrm{nrn} 1824$

5. Rosenberg GA. Neurological diseases in relation to the blood-brain barrier. J Cereb Blood Flow Metab. 2012;32 (7):1139-1151. doi:10.1038/jcbfm.2011.197

6. Daneman R, Prat A. The blood-brain barrier. Cold Spring Harb Perspect Biol. 2015;7(1):a020412. doi:10.1101/cshperspect. a020412

7. Thomsen MS, Routhe LJ, Moos T. The vascular basement membrane in the healthy and pathological brain. J Cereb Blood Flow Metab. 2017;37(10):3300-3317. doi:10.1177/0271678X17722 436

8. Obermeier B, Daneman R, Ransohoff RM. Development, maintenance and disruption of the blood-brain barrier. Nat Med. 2013;19(12):1584-1596. doi:10.1038/nm.3407

9. Wolburg H, Noell S, Fallier-Becker P, Mack AF, WolburgBuchholz K. The disturbed blood-brain barrier in human glioblastoma. Mol Aspects Med. 2012;33(5-6):579-589. doi:10. 1016/j.mam.2012.02.003

10. Obermeier B, Verma A, Ransohoff RM. The blood-brain barrier. Handb Clin Neurol. 2016;133:39-59.

11. van Tellingen O, Yetkin-Arik B, de Gooijer MC, Wesseling P, Wurdinger T, de Vries HE. Overcoming the blood-brain tumor barrier for effective glioblastoma treatment. Drug Resist Updat. 2015;19:1-12. doi:10.1016/j.drup.2015.02.002

12. Watkins S, Robel S, Kimbrough IF, Robert SM, Ellis-Davies G, Sontheimer H. Disruption of astrocyte-vascular coupling and the blood-brain barrier by invading glioma cells. Nat Commun. 2014;5:4196. doi:10.1038/ncomms5196

13. Farin A, Suzuki SO, Weiker M, Goldman JE, Bruce JN, Canoll P. Transplanted glioma cells migrate and proliferate on host brain vasculature: a dynamic analysis. Glia. 2006;53(8):799-808. doi:10.1002/glia.20334

14. Cuddapah VA, Robel S, Watkins S, Sontheimer H. A neurocentric perspective on glioma invasion. Nat Rev Neurosci. 2014;15 (7):455-465. doi:10.1038/nrn3765

15. Montana V, Sontheimer H. Bradykinin promotes the chemotactic invasion of primary brain tumors. $J$ Neurosci. 2011;31 (13):4858-4867. doi:10.1523/JNEUROSCI.3825-10.2011

16. Zagzag D, Amirnovin R, Greco MA, et al. Vascular apoptosis and involution in gliomas precede neovascularization: a novel concept for glioma growth and angiogenesis. Lab Invest. 2000;80 (6):837-849. doi:10.1038/labinvest.3780088

17. Winkler $F$, Kienast $Y$, Fuhrmann $M$, et al. Imaging glioma cell invasion in vivo reveals mechanisms of dissemination and peritumoral angiogenesis. Glia. 2009;57(12):1306-1315. doi:10.1002/glia.20850

18. Winkler EA, Bell RD, Zlokovic BV. Central nervous system pericytes in health and disease. Nat Neurosci. 2011;14 (11):1398-1405. doi:10.1038/nn.2946

19. Abbott NJ, Patabendige AA, Dolman DE, Yusof SR, Begley DJ. Structure and function of the blood-brain barrier. Neurobiol Dis. 2010;37(1):13-25. doi:10.1016/j.nbd.2009.07.030

20. Abbott NJ. Astrocyte-endothelial interactions and blood-brain barrier permeability. J Anat. 2002;200(6):629-638. doi:10.1046/ j.1469-7580.2002.00064.x

21. Jain RK, Di Tomaso E, Duda DG, Loeffler JS, Sorensen AG, Batchelor TT. Angiogenesis in brain tumours. Nat Rev Neurosci. 2007;8(8):610-622. doi:10.1038/nrn2175

22. Shweiki D, Itin A, Soffer D, Keshet E. Vascular endothelial growth factor induced by hypoxia may mediate hypoxia-initiated angiogenesis. Nature. 1992;359(6398):843-845. doi:10.1038/ 359843a0

23. Zlokovic BV. Neurovascular pathways to neurodegeneration in Alzheimer's disease and other disorders. Nat Rev Neurosci. 2011;12(12):723-738. doi:10.1038/nrn3114 
24. Pacioni S, D’Alessandris QG, Buccarelli M, et al. Brain invasion along perivascular spaces by glioma cells: relationship with blood-brain barrier. Cancers (Basel). 2019;12(1):18. doi: $10.3390 /$ cancers 12010018

25. Worthmann H, Tryc AB, Goldbecker A, et al. The temporal profile of inflammatory markers and mediators in blood after acute ischemic stroke differs depending on stroke outcome. Cerebrovasc Dis. 2010;30(1):85-92. doi:10.1159/000314624

26. Bazzoni G, Dejana E. Endothelial cell-to-cell junctions: molecular organization and role in vascular homeostasis. Physiol Rev. 2004;84(3):869-901. doi:10.1152/physrev.00035.2003

27. Markelc B, Bellard E, Sersa G, et al. Increased permeability of blood vessels after reversible electroporation is facilitated by alterations in endothelial cell-to-cell junctions. $J$ Control Release. 2018;276:30-41. doi:10.1016/j.jconrel.2018.02.032

28. Saitou M, Furuse M, Sasaki H, et al. Complex phenotype of mice lacking occludin, a component of tight junction strands. Mol Biol Cell. 2000;11(12):4131-4142. doi:10.1091/mbc.11.12.4131

29. Cardoso FL, Brites D, Brito MA. Looking at the blood-brain barrier: molecular anatomy and possible investigation approaches. Brain Res Rev. 2010;64(2):328-363.

30. Nitta T, Hata M, Gotoh S, et al. Size-selective loosening of the blood-brain barrier in claudin-5-deficient mice. $J$ Cell Biol. 2003;161(3):653-660. doi:10.1083/jcb.200302070

31. Guo J, Cai H, Zheng J, et al. Long non-coding RNA NEAT1 regulates permeability of the blood-tumor barrier via miR-181d5p-mediated expression changes in ZO-1, occludin, and claudin-5. Biochim Biophys Acta Mol Basis Dis. 2017;1863 (9):2240-2254. doi:10.1016/j.bbadis.2017.02.005

32. Zhao L, Wang P, Liu Y, Ma J, Xue Y. miR-34c regulates the permeability of blood-tumor barrier via MAZ-mediated expression changes of ZO-1, occludin, and claudin-5. J Cell Physiol. 2015;230(3):716-731. doi:10.1002/jcp.24799

33. Miao YS, Zhao YY, Zhao LN, et al. MiR-18a increased the permeability of BTB via RUNX1 mediated down-regulation of ZO-1, occludin and claudin-5. Cell Signal. 2015;27(1):156-167. doi:10.1016/j.cellsig.2014.10.008

34. Erickson KK, Sundstrom JM, Antonetti DA. Vascular permeability in ocular disease and the role of tight junctions. Angiogenesis. 2007;10(2):103-117. doi:10.1007/s10456-007-9067-z

35. Liu W, Wang $P$, Shang $C$, et al. Endophilin-1 regulates blood-brain barrier permeability by controlling ZO-1 and occludin expression via the EGFR-ERK1/2 pathway. Brain Res. 2014;1573:17-26. doi:10.1016/j.brainres.2014.05.022

36. Chen L, Liu W, Wang $\mathrm{P}$, et al. Endophilin-1 regulates blood-brain barrier permeability via EGFR-JNK signaling pathway. Brain Res. 2015;1606:44-53. doi:10.1016/j.brainres. 2015.02.032

37. Yang S, Jin H, Zhao Z. An ECV304 monoculture model for permeability assessment of blood-brain barrier. Neurol Res. 2018;40(2):117-121. doi:10.1080/01616412.2017.1398882

38. Neuhaus W, Wirth M, Plattner VE, Germann B, Gabor F, Noe CR. Expression of Claudin-1, Claudin-3 and Claudin-5 in human blood-brain barrier mimicking cell line ECV304 is inducible by glioma-conditioned media. Neurosci Lett. 2008;446(2-3):59-64. doi:10.1016/j.neulet.2008.09.025

39. Neuhaus W, Germann B, Plattner VE, Gabor F, Wirth M, Noe CR. Alteration of the glycocalyx of two blood-brain barrier mimicking cell lines is inducible by glioma conditioned media. Brain Res. 2009;1279:82-89. doi:10.1016/j.brainres. 2009.05.010

40. Lin M, Zhu L, Wang J, Xue Y, Shang X. miR-424-5p maybe regulate blood-brain barrier permeability in a model in vitro with Abeta incubated endothelial cells. Biochem Biophys Res Commun. 2019;517(3):525-531. doi:10.1016/j.bbrc.2019.07 075
41. Liebner S, Fischmann A, Rascher G, et al. Claudin-1 and claudin-5 expression and tight junction morphology are altered in blood vessels of human glioblastoma multiforme. Acta Neuropathol. 2000;100(3):323-331. doi:10.1007/s004010000180

42. Sorokin L. The impact of the extracellular matrix on inflammation. Nat Rev Immunol. 2010;10(10):712-723. doi:10. $1038 /$ nri2852

43. Wu C, Ivars F, Anderson P, et al. Endothelial basement membrane laminin alpha5 selectively inhibits $\mathrm{T}$ lymphocyte extravasation into the brain. Nat Med. 2009;15(5):519-527. doi:10.1038/ nm.1957

44. Song J, Zhang X, Buscher K, et al. Endothelial basement membrane laminin 511 contributes to endothelial junctional tightness and thereby inhibits leukocyte transmigration. Cell Rep. 2017;18 (5):1256-1269. doi:10.1016/j.celrep.2016.12.092

45. Savettieri G, Di Liegro I, Catania C, et al. Neurons and ECM regulate occludin localization in brain endothelial cells. Neuroreport. 2000;11(5):1081-1084. doi:10.1097/00001756200004070-00035

46. Yao Y, Chen ZL, Norris EH, Strickland S. Astrocytic laminin regulates pericyte differentiation and maintains blood brain barrier integrity. Nat Commun. 2014;5(1):3413. doi:10.1038/ ncomms 4413

47. Xhima K, Weber-Adrian D, Silburt J. Glutamate induces blood-brain barrier permeability through activation of N-MethylD-Aspartate receptors. J Neurosci. 2016;36(49):12296-12298. doi:10.1523/JNEUROSCI.2962-16.2016

48. Vazana U, Veksler R, Pell GS, et al. Glutamate-mediated blood-brain barrier opening: implications for neuroprotection and drug delivery. $J$ Neurosci. 2016;36(29):7727-7739. doi:10.1523/JNEUROSCI.0587-16.2016

49. Mendes B, Marques C, Carvalho I, et al. Influence of glioma cells on a new co-culture in vitro blood-brain barrier model for characterization and validation of permeability. Int J Pharm. 2015;490 (1-2):94-101. doi:10.1016/j.ijpharm.2015.05.027

50. Shimizu F, Sano Y, Maeda T, et al. Peripheral nerve pericytes originating from the blood-nerve barrier expresses tight junctional molecules and transporters as barrier-forming cells. J Cell Physiol. 2008;217(2):388-399. doi:10.1002/jcp.21508

51. Sa-Pereira I, Brites D, Brito MA. Neurovascular unit: a focus on pericytes. Mol Neurobiol. 2012;45(2):327-347. doi:10.1007/ s12035-012-8244-2

52. Kim JA, Tran ND, Li Z, Yang F, Zhou W, Fisher MJ. Brain endothelial hemostasis regulation by pericytes. J Cereb Blood Flow Metab. 2006;26(2):209-217. doi:10.1038/sj.jcbfm.9600 181

53. Armulik A, Genove G, Mae M, et al. Pericytes regulate the blood-brain barrier. Nature. 2010;468(7323):557-561. doi:10. 1038/nature09522

54. Bernacki J, Dobrowolska A, Nierwinska K, Malecki A. Physiology and pharmacological role of the blood-brain barrier. Pharmacol Rep. 2008;60(5):600-622.

55. Bell RD, Winkler EA, Sagare AP, et al. Pericytes control key neurovascular functions and neuronal phenotype in the adult brain and during brain aging. Neuron. 2010;68(3):409-427. doi:10.10 16/j.neuron.2010.09.043

56. Wang Y, Pan L, Moens CB, Appel B. Notch3 establishes brain vascular integrity by regulating pericyte number. Development. 2014;141(2):307-317. doi:10.1242/dev.096107

57. Caspani EM, Crossley PH, Redondo-Garcia C, Martinez S. Glioblastoma: a pathogenic crosstalk between tumor cells and pericytes. PLoS One. 2014;9(7):e101402. doi:10.1371/journal. pone. 0101402

58. Engelhardt S, Patkar S, Ogunshola OO. Cell-specific blood-brain barrier regulation in health and disease: a focus on hypoxia. $\mathrm{Br}$ J Pharmacol. 2014;171(5):1210-1230. 
59. Jackson S, ElAli A, Virgintino D, Gilbert MR. Blood-brain barrier pericyte importance in malignant gliomas: what we can learn from stroke and Alzheimer's disease. Neuro-Oncology. 2017;19 (9):1173-1182. doi:10.1093/neuonc/nox058

60. Shimizu F, Sano Y, Saito K, et al. Pericyte-derived glial cell line-derived neurotrophic factor increase the expression of claudin-5 in the blood-brain barrier and the blood-nerve barrier. Neurochem Res. 2012;37(2):401-409. doi:10.1007/s11064-0110626-8

61. Yi D, Xiang W, Zhang Q, et al. Human glioblastoma-derived mesenchymal stem cell to pericytes transition and angiogenic capacity in glioblastoma microenvironment. Cell Physiol Biochem. 2018;46(1):279-290. doi:10.1159/000488429

62. Zhang Q, Yi DY, Xue BZ, et al. CD90 determined two subpopulations of glioma-associated mesenchymal stem cells with different roles in tumour progression. Cell Death Dis. 2018;9(11):1101. doi:10.1038/s41419-018-1140-6

63. Maugeri R, Schiera G, Di Liegro CM, Fricano A, Iacopino DG, Di Liegro I. Aquaporins and brain tumors. Int J Mol Sci. 2016;17 (7):1029. doi:10.3390/ijms17071029

64. Stokum JA, Kurland DB, Gerzanich V, Simard JM. Mechanisms of astrocyte-mediated cerebral edema. Neurochem Res. 2015;40 (2):317-328. doi:10.1007/s11064-014-1374-3

65. Warth A, Kroger S, Wolburg H. Redistribution of aquaporin-4 in human glioblastoma correlates with loss of agrin immunoreactivity from brain capillary basal laminae. Acta Neuropathol. 2004;107(4):311-318. doi:10.1007/s00401-003-0812-0

66. Noell S, Fallier-Becker P, Beyer C, Kroger S, Mack AF, Wolburg H. Effects of agrin on the expression and distribution of the water channel protein aquaporin-4 and volume regulation in cultured astrocytes. Eur J Neurosci. 2007;26(8):2109-2118. doi:10.1111/j.1460-9568.2007.05850.x

67. Noell S, Fallier-Becker P, Deutsch U, Mack AF, Wolburg H. Agrin defines polarized distribution of orthogonal arrays of particles in astrocytes. Cell Tissue Res. 2009;337(2):185-195. doi:10.1007/s00441-009-0812-z

68. Zhou J, Kong H, Hua X, Xiao M, Ding J, Hu G. Altered blood-brain barrier integrity in adult aquaporin-4 knockout mice. Neuroreport. 2008;19(1):1-5. doi:10.1097/WNR.0b013e3 $282 \mathrm{f} 2 \mathrm{~b} 4 \mathrm{eb}$

69. Saadoun S, Tait MJ, Reza A, et al. AQP4 gene deletion in mice does not alter blood-brain barrier integrity or brain morphology. Neuroscience. 2009;161(3):764-772. doi:10.1016/j.neuroscience. 2009.03.069

70. Zhang Y, Xu K, Liu Y, et al. Increased cerebral vascularization and decreased water exchange across the blood-brain barrier in aquaporin-4 knockout mice. PLoS One. 2019;14(6):e0218415. doi:10.1371/journal.pone.0218415

71. Parat M-O, Riggins GJ. Caveolin-1, caveolae, and glioblastoma. Neuro-Oncology. 2012;14(6):679-688. doi:10.1093/neuonc/nos 079

72. Cassoni P, Senetta R, Castellano I, et al. Caveolin-1 expression is variably displayed in astroglial-derived tumors and absent in oligodendrogliomas: concrete premises for a new reliable diagnostic marker in gliomas. Am J Surg Pathol. 2007;31(5):760-769. doi:10.1097/01.pas.0000213433.1474 $0.5 \mathrm{~d}$

73. Ronaldson PT, Bendayan M, Gingras D, Piquette-Miller M, Bendayan R. Cellular localization and functional expression of P-glycoprotein in rat astrocyte cultures. J Neurochem. 2004;89 (3):788-800. doi:10.1111/j.1471-4159.2004.02417.x

74. Machein MR, Kullmer J, Fiebich BL, Plate KH, Warnke PC. Vascular endothelial growth factor expression, vascular volume, and, capillary permeability in human brain tumors. Neurosurgery. 1999;44(4):732-740; discussion 740-731. doi:10.1097/00006123199904000-00022
75. Cao Y, Sundgren PC, Tsien CI, Chenevert TT, Junck L. Physiologic and metabolic magnetic resonance imaging in gliomas. J Clin Oncol. 2006;24(8):1228-1235. doi:10.1200/JCO.2005.04.7233

76. Sarkaria JN, Hu LS, Parney IF, et al. Is the blood-brain barrier really disrupted in all glioblastomas? A critical assessment of existing clinical data. Neuro-Oncology. 2018;20(2):184-191. doi:10.1093/neuonc/nox 175

77. Dhermain FG, Hau P, Lanfermann H, Jacobs AH, van den Bent MJ. Advanced MRI and PET imaging for assessment of treatment response in patients with gliomas. Lancet Neurol. 2010;9(9):906-920. doi:10.1016/S1474-4422(10)70181-2

78. Liu HL, Hua MY, Chen PY, et al. Blood-brain barrier disruption with focused ultrasound enhances delivery of chemotherapeutic drugs for glioblastoma treatment. Radiology. 2010;255(2):4 15-425. doi:10.1148/radiol.10090699

79. Ma J, Wang P, Liu Y, Zhao L, Li Z, Xue Y. Kruppel-like factor 4 regulates blood-tumor barrier permeability via $\mathrm{ZO}-1$, occludin and claudin-5. J Cell Physiol. 2014;229(7):916-926. doi:10.1002/ jcp. 24523

80. Hersh DS, Wadajkar AS, Roberts N, et al. Evolving drug delivery strategies to overcome the blood brain barrier. Curr Pharm Des. 2016;22(9):1177-1193. doi:10.2174/1381612822666151221150733

81. Emerich DF, Dean RL, Osborn C, Bartus RT. The development of the bradykinin agonist labradimil as a means to increase the permeability of the blood-brain barrier: from concept to clinical evaluation. Clin Pharmacokinet. 2001;40(2):105-123. doi:10.216 5/00003088-200140020-00003

82. Fredholm BB, IJzerman AP, Jacobson KA, Linden J, Muller CE. International Union of Basic and Clinical Pharmacology. LXXXI. Nomenclature and classification of adenosine receptors-an update. Pharmacol Rev. 2011;63(1):1-34. doi:10.1124/pr.110. 003285

83. Kim DG, Bynoe MS. A2A adenosine receptor regulates the human blood-brain barrier permeability. Mol Neurobiol. 2015;52 (1):664-678. doi:10.1007/s12035-014-8879-2

84. Bynoe MS, Viret C, Yan A, Kim DG. Adenosine receptor signaling: a key to opening the blood-brain door. Fluids Barriers CNS. 2015;12(1):20. doi:10.1186/s12987-015-0017-7

85. Wu J, Zhao D, Wu S, Wang D. Ang-(1-7) exerts protective role in blood-brain barrier damage by the balance of TIMP-1/MMP-9. Eur J Pharmacol. 2015;748:30-36. doi:10.1016/j.ejphar.2014.12.007

86. Li X, Wang X, Xie J, Liang B, Wu J. Suppression of angiotensin-(1-7) on the disruption of blood-brain barrier in rat of brain glioma. Pathol Oncol Res. 2019;25(1):429-435. doi:10.1007/s12253-018-0471-z

87. Zhou W, Chen C, Shi Y, et al. Targeting glioma stem cell-derived pericytes disrupts the blood-tumor barrier and improves chemotherapeutic efficacy. Cell Stem Cell. 2017;21(5):591-603 e594. doi:10.1016/j.stem.2017.10.002

88. Alvarez JI, Dodelet-Devillers A, Kebir H, et al. The Hedgehog pathway promotes blood-brain barrier integrity and CNS immune quiescence. Science. 2011;334(6063):1727-1731. doi:10.1126/ science. 1206936

89. Argaw AT, Gurfein BT, Zhang Y, Zameer A, John GR. VEGFmediated disruption of endothelial CLN-5 promotes blood-brain barrier breakdown. Proc Natl Acad Sci U S A. 2009;106 (6):1977-1982. doi:10.1073/pnas.0808698106

90. Wen L, Tan Y, Dai S, et al. VEGF-mediated tight junctions pathological fenestration enhances doxorubicin-loaded glycolipid-like nanoparticles traversing BBB for glioblastoma-targeting therapy. Drug Deliv. 2017;24(1):1843-1855. doi:10.1080/10717544.20 17.1386731

91. Diaz RJ, Ali S, Qadir MG, De La Fuente MI, Ivan ME, Komotar RJ. The role of bevacizumab in the treatment of glioblastoma. J Neurooncol. 2017;133(3):455-467. doi:10.1007/ s11060-017-2477-x 
92. Fan $\mathrm{CH}$, Lin $\mathrm{WH}$, Ting $\mathrm{CY}$, et al. Contrast-enhanced ultrasound imaging for the detection of focused ultrasound-induced blood-brain barrier opening. Theranostics. 2014;4(10):10 14-1025. doi:10.7150/thno.9575

93. Zhang J, Liu H, Du X, et al. Increasing of blood-brain tumor barrier permeability through transcellular and paracellular pathways by microbubble-enhanced diagnostic ultrasound in a C6 glioma model. Front Neurosci. 2017;11:86. doi:10.3389/ fnins.2017.00086

94. Meng Y, Pople CB, Lea-Banks H, et al. Safety and efficacy of focused ultrasound induced blood-brain barrier opening, an integrative review of animal and human studies. $J$ Control Release. 2019;309:25-36. doi:10.1016/j.jconrel.2019.07.023

95. Chen P-Y, Hsieh H-Y, Huang C-Y, Lin C-Y, Wei K-C, Liu H-L. Focused ultrasound-induced blood-brain barrier opening to enhance interleukin-12 delivery for brain tumor immunotherapy: a preclinical feasibility study. $J$ Transl Med. 2015;13:93. doi:10.1186/s12967-015-0451-y

96. Ting C-Y, Fan C-H, Liu H-L, et al. Concurrent blood-brain barrier opening and local drug delivery using drug-carrying microbubbles and focused ultrasound for brain glioma treatment. Biomaterials. 2012;33(2):704-712. doi:10.1016/j.biomaterials.20 11.09.096

97. Zhang Z, Xia C, Xue Y, Liu Y. Synergistic effect of low-frequency ultrasound and low-dose bradykinin on increasing permeability of the blood-tumor barrier by opening tight junction. J Neurosci Res. 2009;87(10):2282-2289. doi:10.1002/jnr.22061

98. Wang JE, Liu YH, Liu LB, Xia CY, Zhang Z, Xue YX. Effects of combining low frequency ultrasound irradiation with papaverine on the permeability of the blood-tumor barrier. $J$ Neurooncol. 2011;102(2):213-224. doi:10.1007/s11060-010-0321-7

99. Jue TR, McDonald KL. The challenges associated with molecular targeted therapies for glioblastoma. J Neurooncol. 2016;127 (3):427-434. doi:10.1007/s11060-016-2080-6

100. Shergalis A, Bankhead A, Luesakul U, Muangsin N, Neamati N. Current challenges and opportunities in treating glioblastoma. Pharmacol Rev. 2018;70(3):412-445. doi:10. 1124/pr.117.014944

101. Chen L, Zeng $\mathrm{D}, \mathrm{Xu} \mathrm{N}$, et al. Blood-brain barrier- and blood-brain tumor barrier-penetrating peptide-derived targeted therapeutics for glioma and malignant tumor brain metastases. ACS Appl Mater Interfaces. 2019;11(45):41889-41897. doi:10.1021/acsami.9b14046

102. Agarwal S, Manchanda P, Vogelbaum MA, Ohlfest JR, Elmquist WF. Function of the blood-brain barrier and restriction of drug delivery to invasive glioma cells: findings in an orthotopic rat xenograft model of glioma. Drug Metab Dispos. 2013;41 (1):33-39. doi:10.1124/dmd.112.048322

103. Zhao HF, Wang J, Shao W, et al. Recent advances in the use of PI3K inhibitors for glioblastoma multiforme: current preclinical and clinical development. Mol Cancer. 2017;16(1):100. doi:10.1186/s12943-017-0670-3

104. Uchida Y, Ohtsuki S, Katsukura Y, et al. Quantitative targeted absolute proteomics of human blood-brain barrier transporters and receptors. $J$ Neurochem. 2011;117(2):333-345. doi:10.1111/ j.1471-4159.2011.07208.x

105. Oberoi RK, Parrish KE, Sio TT, Mittapalli RK, Elmquist WF, Sarkaria JN. Strategies to improve delivery of anticancer drugs across the blood-brain barrier to treat glioblastoma. NeuroOncology. 2016;18(1):27-36. doi:10.1093/neuonc/nov164
106. Parrish KE, Sarkaria JN, Elmquist WF. Improving drug delivery to primary and metastatic brain tumors: strategies to overcome the blood-brain barrier. Clin Pharmacol Ther. 2015;97(4):336-346. doi:10.1002/cpt.71

107. Karim R, Palazzo C, Evrard B, Piel G. Nanocarriers for the treatment of glioblastoma multiforme: current state-of-the-art. J Control Release. 2016;227:23-37. doi:10.1016/j.jconrel.20 16.02 .026

108. Galstyan A, Markman JL, Shatalova ES, et al. Blood-brain barrier permeable nano immunoconjugates induce local immune responses for glioma therapy. Nat Commun. 2019;10(1):3850. doi:10.1038/s41467-019-11719-3

109. Shi D, Mi G, Shen Y, Webster TJ. Glioma-targeted dual functionalized thermosensitive Ferri-liposomes for drug delivery through an in vitro blood-brain barrier. Nanoscale. 2019;11 (32):15057-15071. doi:10.1039/C9NR03931G

110. Li X, Tsibouklis J, Weng T, et al. Nano carriers for drug transport across the blood-brain barrier. J Drug Target. 2017;25(1):17-28. doi:10.1080/1061186X.2016.1184272

111. Anders CK, Adamo B, Karginova O, et al. Pharmacokinetics and efficacy of PEGylated liposomal doxorubicin in an intracranial model of breast cancer. PLoS One. 2013;8(5):e61359. doi:10.1371/journal.pone.0061359

112. Rehman M, Madni A, Shi D, et al. Enhanced blood brain barrier permeability and glioblastoma cell targeting via thermoresponsive lipid nanoparticles. Nanoscale. 2017;9(40):15434-15440. doi:10.1039/C7NR05216B

113. Mo J, He L, Ma B, Chen T. Tailoring particle size of mesoporous silica nanosystem to antagonize glioblastoma and overcome blood-brain barrier. ACS Appl Mater Interfaces. 2016;8 (11):6811-6825. doi:10.1021/acsami.5b11730

114. Lin $\mathrm{T}$, Zhao $\mathrm{P}$, Jiang $\mathrm{Y}$, et al. Blood-brain-barrier-penetrating albumin nanoparticles for biomimetic drug delivery via albumin-binding protein pathways for antiglioma therapy. ACS Nano. 2016;10(11):9999-10012. doi:10.1021/acsnano.6b04268

115. Fan K, Jia X, Zhou M, et al. Ferritin nanocarrier traverses the blood brain barrier and kills glioma. ACS Nano. 2018;12 (5):4105-4115. doi:10.1021/acsnano.7b06969

116. Kumari S, Ahsan SM, Kumar JM, Kondapi AK, Rao NM. Overcoming blood brain barrier with a dual purpose Temozolomide loaded Lactoferrin nanoparticles for combating glioma (SERP-17-12433). Sci Rep. 2017;7(1):6602. doi:10.1038/ s41598-017-06888-4

117. Rufino-Ramos D, Albuquerque PR, Carmona V, Perfeito R, Nobre RJ, Pereira de Almeida L. Extracellular vesicles: novel promising delivery systems for therapy of brain diseases. $J$ Control Release. 2017;262:247-258. doi:10.1016/j.jconrel.2017.07.001

118. Kolter M, Ott M, Hauer C, Reimold I, Fricker G. Nanotoxicity of poly(n-butylcyano-acrylate) nanoparticles at the blood-brain barrier, in human whole blood and in vivo. $J$ Control Release. 2015;197:165-179. doi:10.1016/j.jconrel.2014.11.005

119. Tang J, Xiong L, Zhou G, et al. Silver nanoparticles crossing through and distribution in the blood-brain barrier in vitro. J Nanosci Nanotechnol. 2010;10(10):6313-6317. doi:10.1166/ jnn.2010.2625

120. Tang W, Fan W, Lau J, Deng L, Shen Z, Chen X. Emerging blood-brain-barrier-crossing nanotechnology for brain cancer theranostics. Chem Soc Rev. 2019;48(11):2967-3014. doi: $10.1039 / \mathrm{C} 8 \mathrm{CS} 00805 \mathrm{~A}$ 


\section{Publish your work in this journal}

Cancer Management and Research is an international, peer-reviewed The manuscript management system is completely online and includes open access journal focusing on cancer research and the optimal use of preventative and integrated treatment interventions to achieve improved a very quick and fair peer-review system, which is all easy to use. Visit http://www.dovepress.com/testimonials.php to read real quotes outcomes, enhanced survival and quality of life for the cancer patient.

from published authors. 